\title{
Selection of sampling points for saturation recovery based myocardial $T_{1}$ mapping
}

\author{
Mehmet Akcakaya ${ }^{1 *}$, Sebastian Weingartner ${ }^{1,2}$, Warren J Manning ${ }^{1,3}$, Reza Nezafat ${ }^{1}$ \\ From 17th Annual SCMR Scientific Sessions \\ New Orleans, LA, USA. 16-19 January 2014
}

\section{Background}

Quantitative myocardial $\mathrm{T}_{1}$ mapping allows assessment of focal and diffuse fibrosis in the myocardium, by sampling the $\mathrm{T}_{1}$ relaxation curve using inversion [1] or saturation recovery (SR) preparation [2] or a combination of both [3], followed by the acquisition of multiple images with different contrasts, which are subsequently fitted to a parametric equation pixel-wise to yield the $T_{1}$ maps. In myocardial $\mathrm{T}_{1}$ mapping, there is a degree of freedom in selecting which points on the relaxation curve are sampled. However, this topic has not been studied. In this study, we sought to develop an estimation theoretic framework for optimal selection of sampling points and characterized the variance of the corresponding $\mathrm{T}_{1}$ estimator for sampling of the SR curve.

\section{Methods}

Based on the signal model, $\mathrm{y}_{\mathrm{k}}=\mathrm{a}\left(1-\mathrm{b} \exp \left(-\mathrm{x}_{\mathrm{k}} / \mathrm{T}_{1}\right)\right)+\mathrm{n}_{\mathrm{k}}$, and the least squares model, we derived the Fisher information matrix [4]. This was used to derive the Bayesian Cramer-Rao bound [4] for the variance of the $\mathrm{T}_{1}$ estimator for $\mathrm{T}_{1}$ values of interest between 950 and $1250 \mathrm{~ms}$ ( $\sim$ pre-contrast myocardium). The bound was evaluated for the SASHA sequence [2] which allows sampling

\begin{tabular}{|c|c|c|c|c|c|c|}
\hline \multirow[b]{2}{*}{ Vial } & \multicolumn{2}{|c|}{ uniformly distributed points } & \multicolumn{4}{|c|}{ proposed point selection } \\
\hline & $\mathrm{T}_{1}{ }^{\text {est }}(\mathrm{ms})$ & $\operatorname{std}\left(T_{1}{ }^{e s t}\right)(m s)$ & $\mathrm{T}_{1}{ }^{\text {est }}(\mathrm{ms})$ & $\operatorname{std}\left(T_{1}{ }^{e s t}\right)(m s)$ & $\begin{array}{l}\text { std wrt. } \\
\text { uniform }\end{array}$ & $\begin{array}{l}\text { theory std } \\
\text { wrt. uniform }\end{array}$ \\
\hline 1 & $1457 \pm 7.7$ & 69.5 & $1456 \pm 7.4$ & 48.4 & 0.69 & 0.71 \\
\hline 2 & $1144 \pm 14.5$ & 56.1 & $1130 \pm 7.1$ & 41.1 & 0.73 & 0.76 \\
\hline 3 & $1151 \pm 11.5$ & 53.3 & $1155 \pm 8.6$ & 43.2 & 0.81 & 0.76 \\
\hline 4 & $729 \pm 10.3$ & 31.3 & $724 \pm 2.1$ & 26.3 & 0.84 & 0.86 \\
\hline 5 & $980 \pm 11.2$ & 34.6 & $981 \pm 10.4$ & 25.2 & 0.73 & 0.78 \\
\hline 6 & $823 \pm 13.3$ & 29.9 & $822 \pm 7.7$ & 24.2 & 0.81 & 0.83 \\
\hline 7 & $1148 \pm 18.4$ & 53.0 & $1144 \pm 8.3$ & 37.8 & 0.71 & 0.76 \\
\hline 8 & $1130 \pm 10.6$ & 56.1 & $1137 \pm 10.5$ & 45.2 & 0.81 & 0.76 \\
\hline 9 & $963 \pm 13.8$ & 50.0 & $962 \pm 6.2$ & 35.5 & 0.71 & 0.79 \\
\hline
\end{tabular}

Figure 1 Results of the phantom imaging over vials with $T_{1}$ values $>700 \mathrm{~ms}$ using the proposed and uniform sampling strategies, where each acquisition was repeated $\mathbf{5}$ times. The ratio of the standard deviation of the $T_{1}$ estimator for each proposed sampling strategy and that of the uniform sampling strategy is reported as "standard deviation (std) with respect to (wrt) uniform." There is a gain in using the proposed point selection strategy, which is significantly different than $1(P<0.001)$. The values match those predicted by theory $(P=0.23)$.

'Medicine, Beth Israel Deaconess Medical Center, Harvard Medical School,

Boston, Massachusetts, USA

Full list of author information is available at the end of the article 


\begin{tabular}{|c|c|c|c|c|c|c|}
\hline \multirow[b]{2}{*}{ subject } & \multirow[b]{2}{*}{ anatomy } & \multicolumn{2}{|c|}{ uniform point selection } & \multicolumn{2}{|c|}{ proposed point selection } & \multirow[b]{2}{*}{$\begin{array}{l}\text { std wrt. } \\
\text { uniform }\end{array}$} \\
\hline & & $\mathrm{T}_{1}^{\text {est }}(\mathrm{ms})$ & $\mathrm{std}\left(\mathrm{T}_{1}{ }^{\mathrm{est}}\right)(\mathrm{ms})$ & $\mathrm{T}_{1}{ }^{\mathrm{est}}(\mathrm{ms})$ & $\operatorname{std}\left(T_{1}{ }^{e s t}\right)(m s)$ & \\
\hline \multirow[t]{2}{*}{1} & myocardium & $1211 \pm 6.4$ & $110.1 \pm 10.3$ & $1201 \pm 9.2$ & $84.0 \pm 8.2$ & 0.76 \\
\hline & blood & $1925 \pm 22.5$ & $166.7 \pm 14.8$ & $1903 \pm 17.2$ & $111.8 \pm 6.9$ & 0.67 \\
\hline \multirow[t]{2}{*}{2} & myocardium & $1242 \pm 56.9$ & $132.9 \pm 32.5$ & $1254 \pm 58.9$ & $101.5 \pm 12.4$ & 0.76 \\
\hline & blood & $1772 \pm 21.6$ & $204.9 \pm 28.9$ & $1779 \pm 48.5$ & $147.4 \pm 6.9$ & 0.72 \\
\hline \multirow[t]{2}{*}{3} & myocardium & $1187 \pm 55.0$ & $117.0 \pm 15.0$ & $1218 \pm 33.8$ & $83.0 \pm 12.0$ & 0.71 \\
\hline & blood & $1787 \pm 33.4$ & $179.7 \pm 23.7$ & $1809 \pm 31.7$ & $138.1 \pm 13.4$ & 0.77 \\
\hline \multirow[t]{2}{*}{4} & myocardium & $1213 \pm 44.3$ & $107.3 \pm 14.9$ & $1207 \pm 28.4$ & $85.0 \pm 4.8$ & 0.79 \\
\hline & blood & $1755 \pm 31.0$ & $161.4 \pm 14.9$ & $1780 \pm 17.2$ & $131.4 \pm 11.5$ & 0.81 \\
\hline \multirow[t]{2}{*}{5} & myocardium & $1168 \pm 48.5$ & $95.3 \pm 6.1$ & $1187 \pm 13.3$ & $76.6 \pm 4.4$ & 0.80 \\
\hline & blood & $1772 \pm 42.7$ & $164.7 \pm 16.9$ & $1761 \pm 20.8$ & $114.2 \pm 9.3$ & 0.69 \\
\hline
\end{tabular}

Figure 2 Results of in-vivo imaging for five healthy subjects using the proposed and uniform sampling strategies, where each acquisition was repeated 5 times. $T_{1}$ est is reported as the mean \pm std of the average $T_{1}$ values in the ROI across 5 scans, as a surrogate for accuracy and inter-scan reproducibility. The std $\left(T_{1}{ }^{\text {est }}\right)$ is reported as the mean \pm std of the std of the $T_{1}$ values in the ROI across 5 scans, as a surrogate for the precision within the scan. Std wrt. uniform is the ratio of the mean values of $\operatorname{std}\left(\mathrm{T}_{1}{ }^{\text {est }}\right)$ using the proposed and uniform point selection, as a surrogate for the percentage gain in precision. The standard deviation of the $T_{1}$ estimator in the myocardium and blood was reduced by $23.6 \%$ and $26.8 \%$ respectively using the proposed approach.

within a heart-beat between $\mathrm{T}_{\min }$ and $\mathrm{T}_{\max }$ with one point at full magnetization recovery $\left(\mathrm{x}_{\mathrm{k}}=\infty\right)$, and minimized over the choice of sampling points $\left\{x_{k}\right\}$ yielding the proposed point selection. Phantom imaging of $\mathrm{NiCl}_{2}$ doped agarose vials was performed to compare the proposed point selection with a uniform distribution of sampling points between $\mathrm{T}_{\min }$ and $\mathrm{T}_{\max }$ [3] using an SSFP sequence with body-coil (NSA $=5$ ) for 11 sampling points. Standard deviation (std) of $\mathrm{T}_{1}$ values within the vials was used as a surrogate for the variance of the estimator. Imaging was also performed on 5 healthy adult subjects ( 4 women, $23.4 \pm 3.3$ years) with a 32 -channel cardiac-coil to verify the gains predicted by the theory. Both proposed and uniform point selection acquisitions were repeated 5 times per subject to average out the effects of noise. ROIs were drawn in the myocardium and the blood. Both the $T_{1}$ estimate (average $T_{1}$ values in the ROI) and the std of the estimator (std of $T_{1}$ values in the ROI) are reported as mean \pm std across 5 scans.

\section{Results}

The point selection yielded a tri-modal distribution of points: 4 at $\mathrm{T}_{\min }, 6$ at $\mathrm{T}_{\max }, 1$ at $\infty$, with a theoretical gain in std of $24 \%$ compared to uniform selection. Figure 1 shows the results of phantom imaging for $T_{1}$ values $>700 \mathrm{~ms}$, indicating a good match between theory and experiment. Figure 2 depicts the measurements from the in-vivo data, averaged over five scans. Overall, there was a $23.6 \%$ and $26.8 \%$ reduction in the std of the $\mathrm{T}_{1}$ maps in the myocardium and blood respectively using the proposed approach.

\section{Conclusions}

The proposed framework allows for choosing the location of points on the $T_{1}$ relaxation curve to achieve higher levels of precision without increasing the scan time.

\section{Funding}

NIH:K99HL111410-01; R01EB008743-01A2.

\section{Authors' details}

'Medicine, Beth Israel Deaconess Medical Center, Harvard Medical School, Boston, Massachusetts, USA. ${ }^{2}$ Computer Assisted Clinical Medicine, University Medical Center Mannheim, Heidelberg University, Mannheim, Germany.

${ }^{3}$ Radiology, Beth Israel Deaconess Medical Center, Harvard Medical School, Boston, Massachusetts, USA.

Published: 16 January 2014

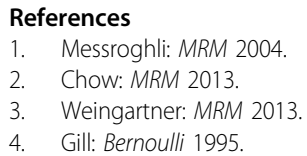

